\title{
Carbon Nitride Functionalized with Sb Resulting in High Photocatalytic Activity
}

Luhong Zhang ${ }^{1,2}$, Zhengyuan Jin ${ }^{3}$, Jiyu Huang ${ }^{1}$, Yiyue Zhang ${ }^{1}$, Shaolong Huang ${ }^{4,5}$, Zhiliang Wang ${ }^{6}$, Yu-Jia Zeng ${ }^{1 *}$, Victor Malgras ${ }^{7}$, Shuangchen Ruan ${ }^{1,3}$ and Yusuke Yamauchi ${ }^{2,6,7 *}$

1 College of Physics and Optoelectronic Engineering, Shenzhen University, Shenzhen, 518060, P. R. China

2 Australian Institute for Bioengineering and Nanotechnology (AIBN), The University of Queensland, Brisbane, QLD 4072, Australia

3 Ctr Adv Mat Diagnost Technol, Shenzhen Technol University, Shenzhen, 518118, P. R. China

4 College of Environmental Science and Engineering, Nankai University, Tianjin, 300350, P. R. China

5 Tianjin Wanfeng Environmental Protection Technology Co., Ltd, Tianjin, 300308, P. R. China

6 School of Chemical Engineering, The University of Queensland, Brisbane, QLD 4072, Australia

7 JST-ERATO Yamauchi Materials Space-Tectonics Project and International Center for Materials Nanoarchitectonics (WPI-MANA), National Institute for Materials Science (NIMS), Tsukuba, 305-044, Japan

E-mails: yjzeng@szu.edu.cn and y.yamauchi@uq.edu.au 


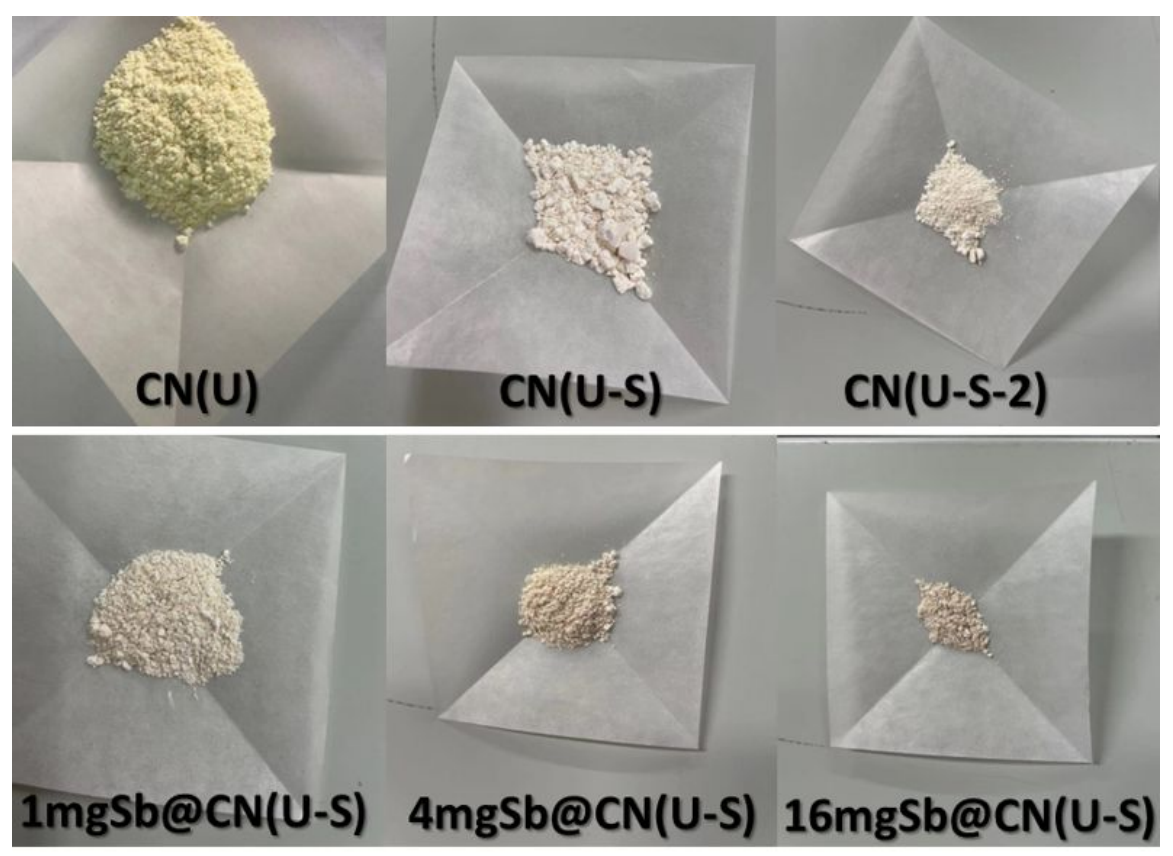

Figure S1. Sample colors of CN(U), CN(U-S), CN(U-S-2), 1mgSb@CN(U-S),4mgSb@CN(U-S) and 16mgSb@CN(U-S).
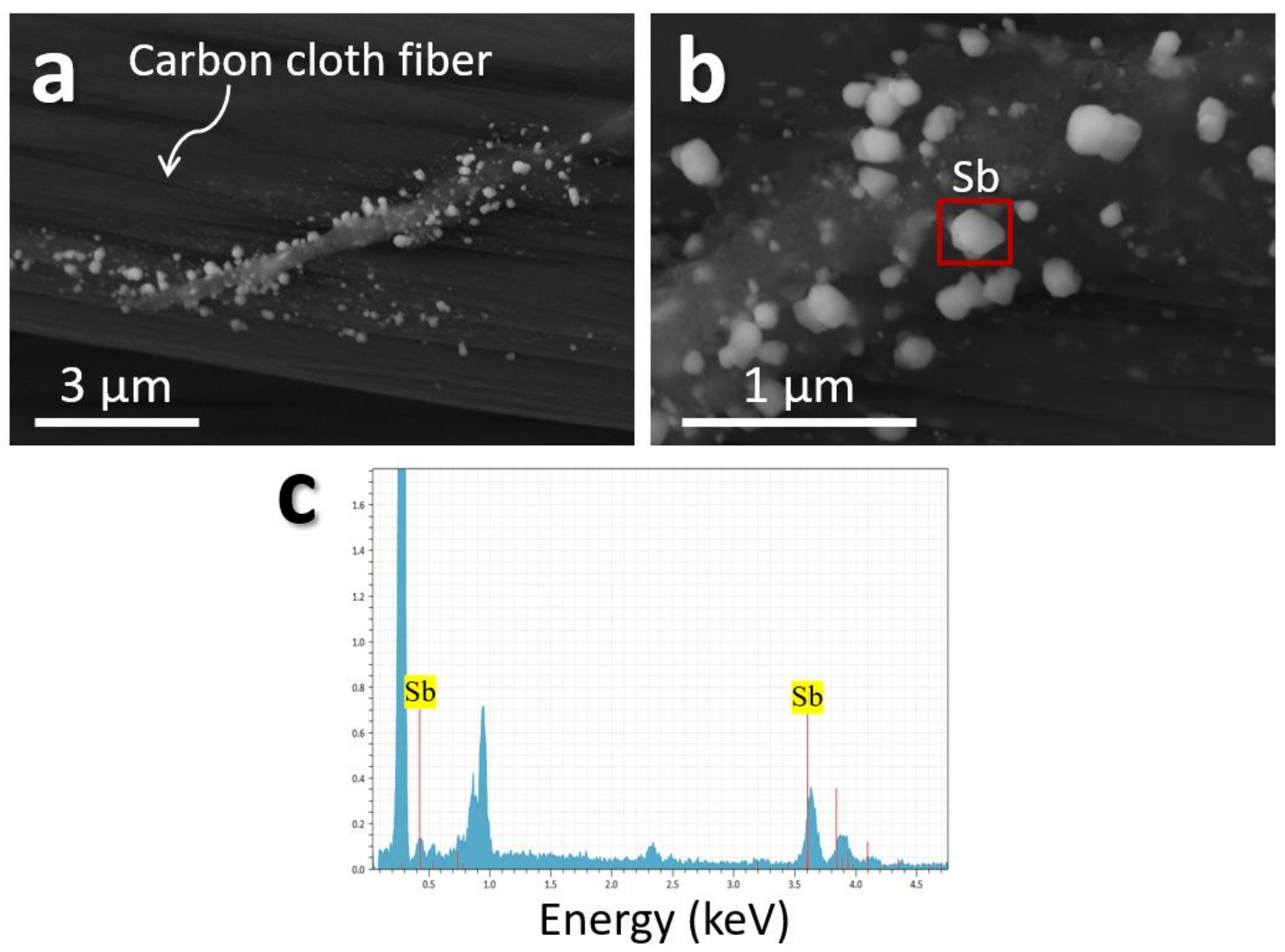

Figure S2. (a, b) SEM images of the Sb-loaded carbon cloth fiber and (c) EDX for the sample. 


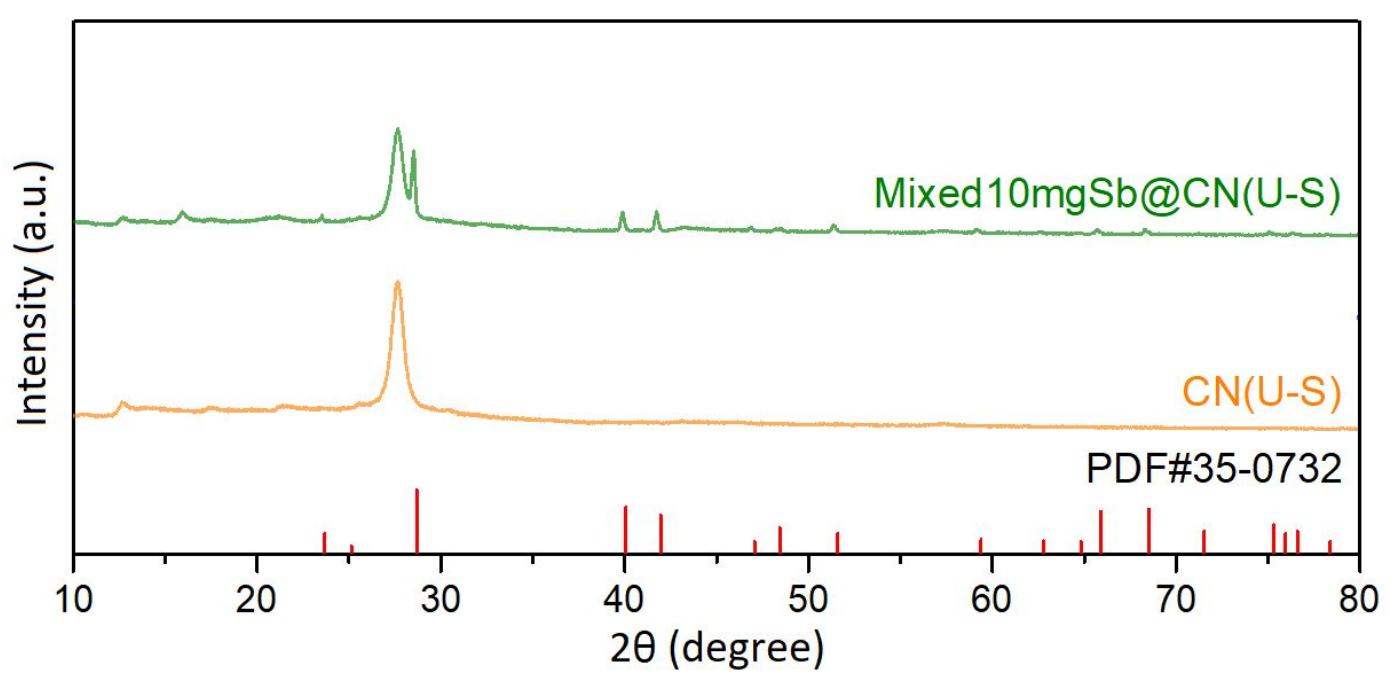

Figure S3. Wide-angle XRD patterns of CN(U-S) and mixed 10mgSb@CN(U-S) which was prepared by directly mixing $\mathrm{Sb}$ powders with $\mathrm{g}-\mathrm{C}_{3} \mathrm{~N}_{4} \mathrm{CN}(\mathrm{U})$ before sealed-tube calcination.

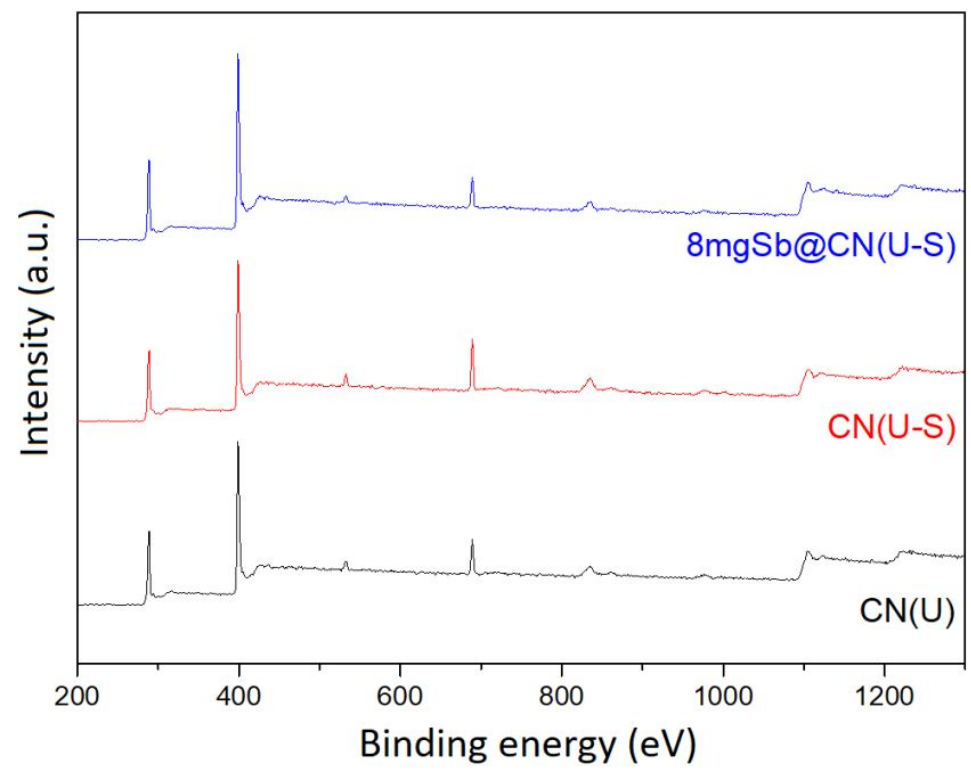

Figure S4. XPS survey spectra for CN(U), CN(U-S) and 8mgSb@CN(U-S). 

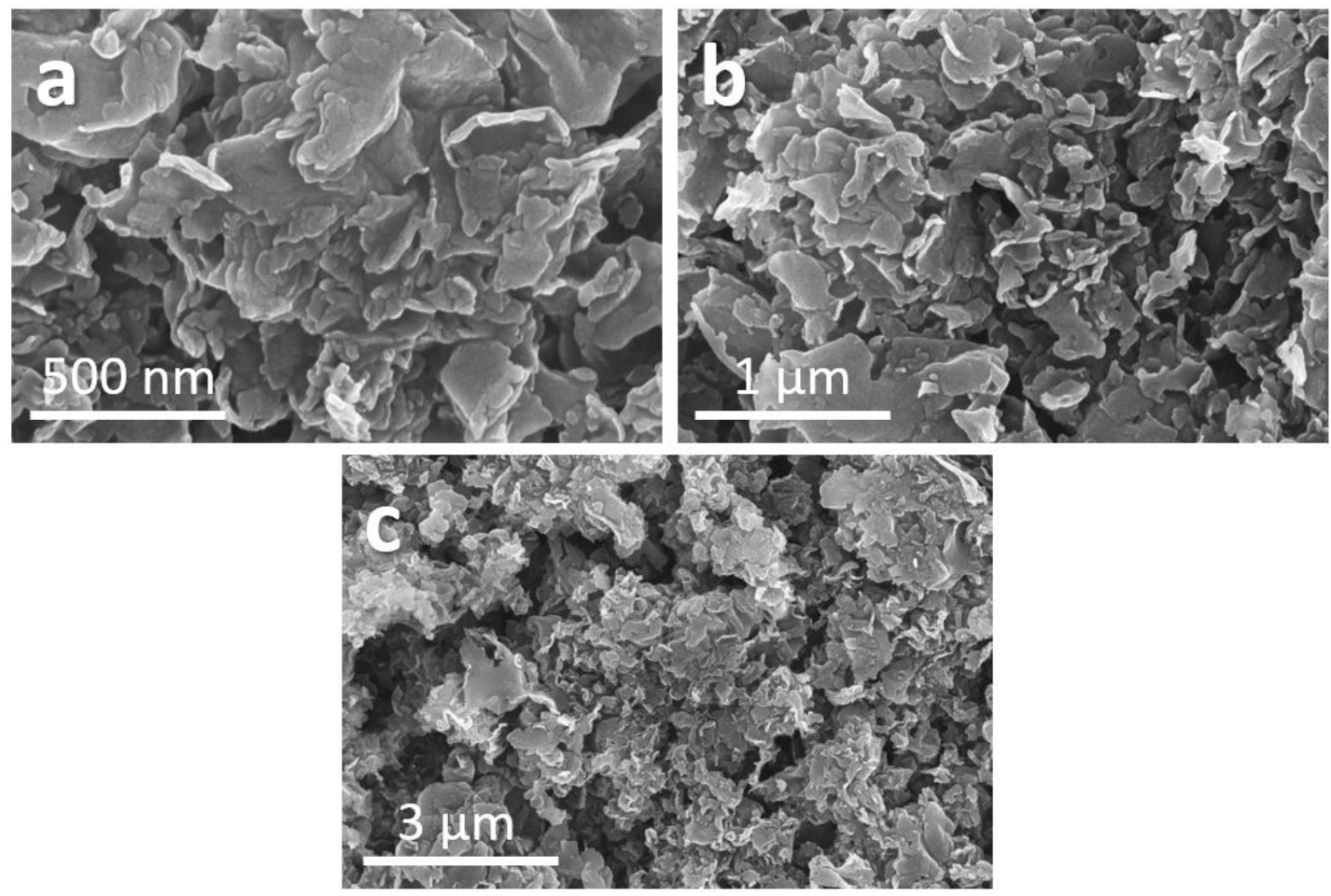

Figure S5. SEM images of 16mgSb@CN(U-S) at different magnifications.
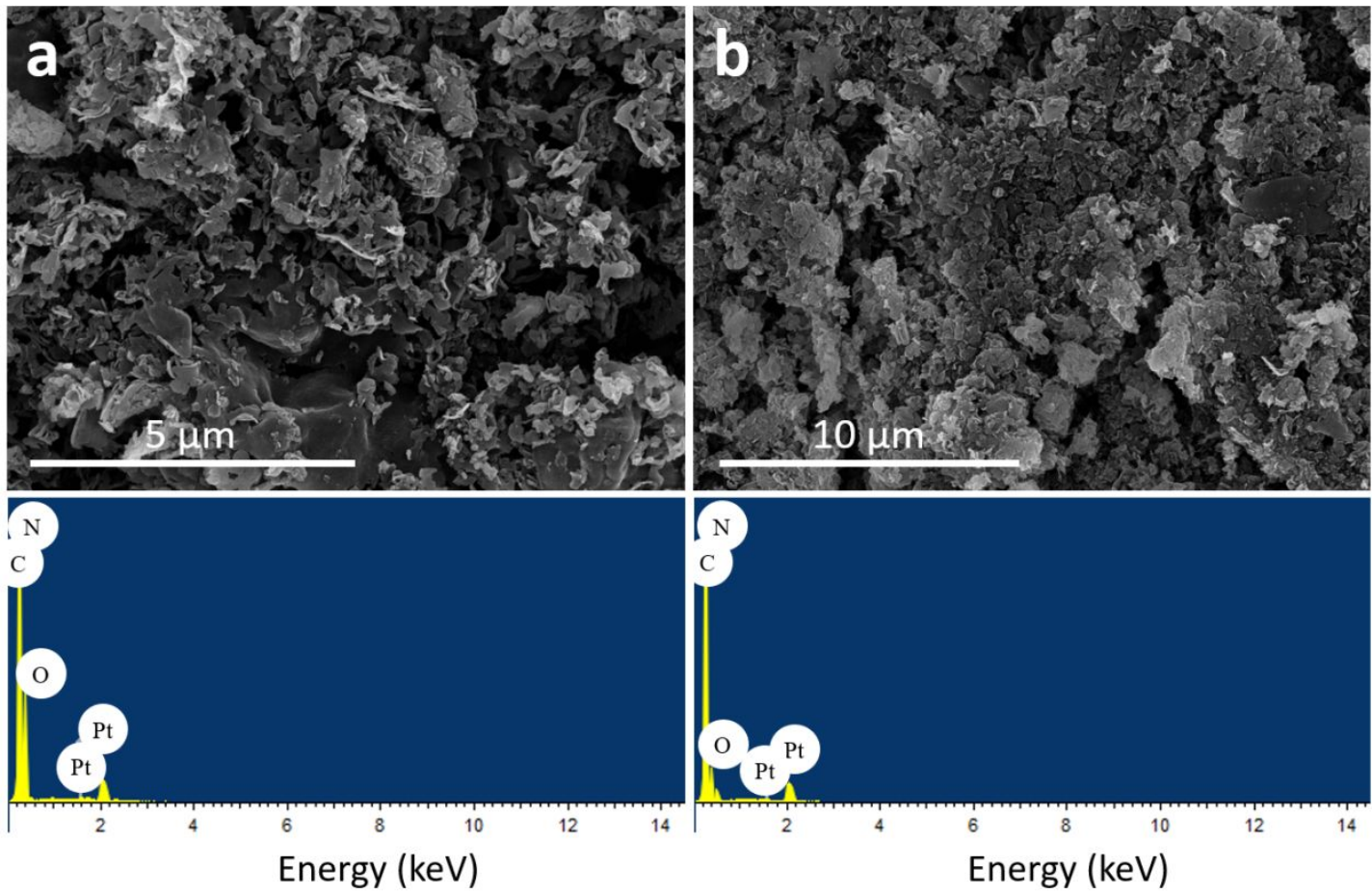

Figure S6. SEM images and the corresponding EDX spectra for (a) 8mgSb@CN(U-S) and (b) 16mgSb@CN(U-S). 
Figure S7. HAADF images of 2mgSb@CN(U-S). The atomic-level Sb species are dispersed evenly in the form of tiny bright dots. The white dots $\mathrm{Sb}$ species are more presented in a continuous line on the edge of g$\mathrm{C}_{3} \mathrm{~N}_{4}$ matrix. 